\title{
Influence Factors on the Formation of Acrylamide in the Amino Acid/Sugar Chemical Model System
}

\author{
Xiao Wang, Liheng Xu* \\ Department of Quality and Safety Engineering, China Jiliang University, Hangzhou, China \\ *Corresponding author: xlheng@cjlu.edu.cn
}

Received June 22, 2014; Revised June 26, 2014; Accepted July 01, 2014

\begin{abstract}
Acrylamide, a potential carcinogen and neurotoxin, could be formed during the heat processing of carbohydrate-rich foods under high temperatures. Maillard reaction between reducing sugars and amino acids is the main pathway to form acrylamide. All of the types of reducing sugars and amino acids as well as the addition of vitamins may influence the formation of acrylamide. To explore the influence of reducing sugars, amino acids and the addition of vitamins on acrylamide formation, different chemical model systems were designed and studied in this work. The results showed that the largest amount of acrylamide was produced in the asparagine/glucose model system. L-glycine, L-glutamic acid and L-cysteine caused different levels of reduction in the amounts of acrylamide produced in the asparagine/glucose model systems. Vitamin $C$ and Vitamin $B_{1}$ strongly reduced the formation of acrylamide by more than $60 \%$ at the appropriate adding dosage of approximately $1 \%$, whereas Vitamin $\mathrm{B}_{2}$ and Vitamin $B_{5}$ only reduced the formation of acrylamide by $20-30 \%$ at the adding dosage of approximately $1 \%$. However, Vitamin $B_{2}$ promoted the formation of acrylamide at adding dosages greater than $1 \%$.
\end{abstract}

Keywords: acrylamide, amino acids, reducing sugars, vitamins, food safety, chemical model system

Cite This Article: Xiao Wang, and Liheng Xu, "Influence Factors on the Formation of Acrylamide in the Amino Acid/Sugar Chemical Model System.” Journal of Food and Nutrition Research, vol. 2, no. 7 (2014): 344348. doi: 10.12691/jfnr-2-7-3.

\section{Introduction}

Food safety is a stake major issue, related to people's health and safety of life as well as economic development and social harmony. In recent years, the poisonous and harmful residues and contaminants in food, especially the hazardous substance produced in the food processing have been in hot research focus all over the world. In April 2002, researchers at the Swedish National Food Administration and Stockholm University for the first time found relatively high levels of acrylamide (AA) in the fried or baked potatoes and plant-based foods [1]. After the report was published, it immediately caused widespread concern in the world food industry. Many confirmatory experiments $[2,3,4]$ have been performed by other research groups from academic, industrial and official laboratories. These investigations caused considerable concern as AA has been regarded as a compound that has neurotoxicity, reproductive toxicity, genetic toxicity and carcinogenicity in animals $[5,6]$.

It is now generally accepted that the Maillard reaction between reducing sugars and carbonyl compounds at high temperatures is the key pathway for the formation of AA in foods $[7,8,9,10,11]$. Reducing sugars and amino acids involved in the Maillard reaction provide the molecular skeleton of AA. A large number of reports [12,13,14,15] have evaluated the effect of amino acids on AA, but few reports can be found on the effect of amino acid mixtures on AA formation. Meanwhile, the type of reducing sugar is also an important factor that influences the formation of AA.

Many additives have been reported to influence AA formation. Several of these additives have inhibition effects, such as organic acids [16,17], chlorates [17,18,19], phenolic compounds [20,21], and plant extract [22,23]. In contrast, several of these additives have promoting effects, such as sucrose [24] and butylated hydroxytoluene [25]. Vitamins have traditionally been among the most widely applied chemical agents to enhance the nutritional values of food products. Moreover, flour itself contains many vitamins, including Vitamin $\mathrm{B}_{1}$, Vitamin $\mathrm{B}_{2}$ and Vitamin $\mathrm{B}_{5}$, in addition to trace amounts of vitamin $\mathrm{C}$. However, there is no scientific consensus about the influence of vitamins on AA formation in the amino acid/sugar reaction system.

Taking into consideration all described above, the present study aimed to investigate the influence factors on AA formation in the amino acid/sugar model system, especially the mixing of amide acids, the types of reducing sugars and the addition of vitamins. The tested reducing sugars included L-glucose (GLU), fructose (Fru), Dxylose (Xyl), maltose (Mal), D-anhydrous lactose (Lac) and galactose (Gal). The tested amino acids included Lasparagine (Asn), L-glycine (Gly), L-glutamic acid (Glu) and L-cysteine (Cys). The tested vitamins included Lascorbic acid (vitamin $\mathrm{C}$; VC), thiamin (vitamin $\mathrm{B}_{1}$; $\mathrm{VB}_{1}$ ), riboflavin (vitamin $\mathrm{B}_{2} ; \mathrm{VB}_{2}$ ) and calcium pantothenate (vitamin $\mathrm{B}_{5} ; \mathrm{VB}_{5}$ ). 


\section{Material and Methods}

\subsection{Reagents and chemicals}

All solvents used were of HPLC grade, and the other chemicals were of analytical grade. Acrylamide (purity > 99\%) and HPLC-grade methanol were obtained from J\&K
Technology Co., Ltd. (Beijing, China). L-asparagine, Lglycine, L-glutamic acid, L-cysteine, L-glucose, fructose, D-xylose, maltose, D-anhydrous lactose, galactose, Lascorbic acid, thiamin, riboflavin and calcium pantothenate were obtained from Hangzhou Mike Chemical Instrument Co., Ltd. (Hangzhou, China). The chemical structure formulas of the testing reducing sugars, amino acids and vitamins are listed in Table 1.

Table 1. Chemical structure formulas of the tested reducing sugars and amino acids

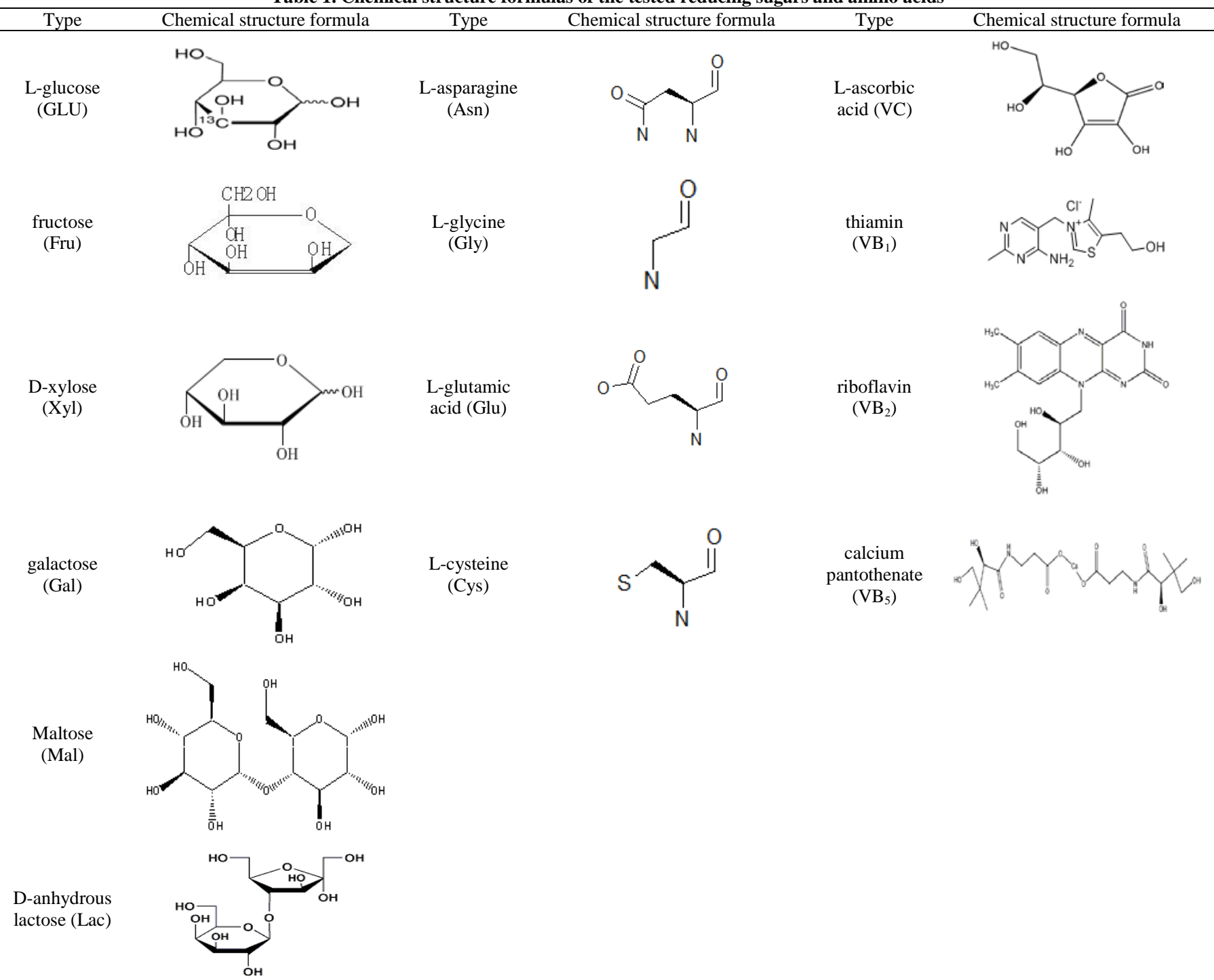

\subsection{Apparatus}

An oven (Midea, China) was used in this experiment. The extracts were prepared with a solid phase extraction (Visiprep TM DL, Supelco, United States) with a HC-C18 solid phase extraction small column $(500 \mathrm{mg} / 3 \mathrm{~mL}$; Shanghai spectrum). Chromatographic analysis was performed on a high performance liquid chromatograph (Agilent 1260).

\subsection{Chemical Model Reactions of AA Formation}

Chemical model reactions were performed according to the method of [26] with minor modifications described by [27], and all of the experiments were performed in triplicate.

The amino acid/reducing-sugar model systems were prepared as follows: $0.1 \mathrm{mmol}$ reducing sugars (GLU, Fru,
Xyl, Mal, Lac or Gal) and $0.1 \mathrm{mmol}$ amino acids (Asn, Gly, Glu or Cys) were mixed with $3 \mathrm{~mL}$ of purified water. The reaction vials were heated in the oven at $160^{\circ} \mathrm{C}$ for 30 min, and the reaction mixtures were then diluted with purified water to $5 \mathrm{~mL}$ after they were cooled. The C18 solid phase extraction small columns were activated by 2 $\mathrm{mL}$ of methanol and $2 \mathrm{~mL}$ of water. The combined solvent (1 $\mathrm{mL}$ ) was injected into the small column, and the effluent was discarded. The remaining portion of the combined solvent was also injected into the small column, and the effluent was collected for HPLC.

The dynamic Asn/reducing sugar model systems were prepared as follows: $0.1 \mathrm{mmol}$ Asn and $0.1 \mathrm{mmol}$ reducing sugars (GLU, Fru or Xyl) were mixed with $3 \mathrm{~mL}$ of purified water, and the reaction vials were heated in the oven at $160{ }^{\circ} \mathrm{C}$ for a certain gradient of time (5-50 min). Then, the reaction mixtures were treated as described above. 
The mixed amino acid/GLU model systems were prepared as follows: $0.1 \mathrm{mmol}$ amino acids were added to the Asn/GLU model system. The reaction vials were heated in the oven at $160^{\circ} \mathrm{C}$ for $30 \mathrm{~min}$, and the reaction mixtures were then treated as described above.

The vitamin/Asn/GLU chemical model systems were prepared. (1) The four types of water-soluble vitamins (VC, $\mathrm{VB}_{1}, \mathrm{VB}_{2}$ and $\mathrm{VB}_{5}$ ) were either added $(1 \%)$ or not to the blank sample based on the Asn/GLU model system, and the reaction vials were heated in the oven at $160^{\circ} \mathrm{C}$ for $30 \mathrm{~min}$. The reaction mixtures were then treated as described above. (2) The four types of water-soluble vitamins ( $\mathrm{VC}, \mathrm{VB}_{1}, \mathrm{VB}_{2}, \mathrm{VB}_{5}$ ) were either added (0.1-5\%) or not to the blank sample based on the Asn/GLU model system, and the reaction vials were heated in the oven at $160^{\circ} \mathrm{C}$ for $30 \mathrm{~min}$. The reaction mixtures were then treated as described above.

\subsection{Analysis of AA by HPLC}

The analysis method of AA was performed as described by [28] and [29]. This method was confirmed by HPLC in our study. The determination of AA formation was performed using a HPLC system (Agilent Technologies Co., USA) equipped with automatic sampler and diode array detector (DAD), and a reversed ZORBAX SBC18ODSC18 $(4.6 \times 150 \mathrm{~mm}, 5 \mu \mathrm{m}$, Agilent Technologies Co., USA). Solution ( $15 \mu \mathrm{L})$ was injected into the column and eluted isocratically at $30^{\circ} \mathrm{C}$ with $10 \%$ methanol in water at a flow rate of $0.5 \mathrm{~mL} / \mathrm{min}$. The post running time was $15 \mathrm{~min}$ and the detection wavelength was $210 \mathrm{~nm}$. AA was quantified by external calibration, in which the concentrations of AA standard solutions ranged from 0.01 to $1.0 \mu \mathrm{g} / \mathrm{mL}$.

\section{Results and Discussion}

\subsection{Effects of the Type of Reducing Sugars on AA Formation}

Six types of reducing sugars, including four types of monosaccharide and two types of disaccharide, were examined for their effect on the formation of AA with three common amino acids in this experiment.

On one hand, different types of reducing sugars have different capacities to produce AA with amino acid. As indicated by [8], Asn is the main amino acid that reacts with reducing sugars to produce AA. As shown in Figure 1 , different amounts of AA were produced in different Asn/reducing sugar model systems. The abilities of reducing sugars to produce AA with Asn were ranked as follows: GLU $(0.1103 \mu \mathrm{g} / \mathrm{mL})>$ Fru $(0.0963 \mu \mathrm{g} / \mathrm{mL})>$ Xyl $(0.0459 \mu \mathrm{g} / \mathrm{mL})>$ Gal $(0.0449 \mu \mathrm{g} / \mathrm{mL})>$ Mal $(0.03751 \mu \mathrm{g} / \mathrm{mL})>\operatorname{Lac}(0.0175 \mu \mathrm{g} / \mathrm{mL})$. This order indicates that monosaccharide may have a stronger capability to produce AA with Asn. In addition, different amounts of AA were produced in different Gly/reducing sugar model systems and Glu/reducing sugar model systems.

On the other hand, the same type of reducing sugars has different capacity to produce AA with different types of amino acids. In this experiment, GLU was selected and used, and three types of reducing sugar were examined for their effect on the formation of AA. As shown in Figure 1, different amounts of AA were produced in different amino acid/GLU model systems. The abilities of GLU to produce AA with different types of amino acids were ranked as follows: Asn $(0.1154 \mu \mathrm{g} / \mathrm{mL})>$ Gly $(0.0282 \mu \mathrm{g} / \mathrm{mL})>$ Glu $(0.0101 \mu \mathrm{g} / \mathrm{mL})$.

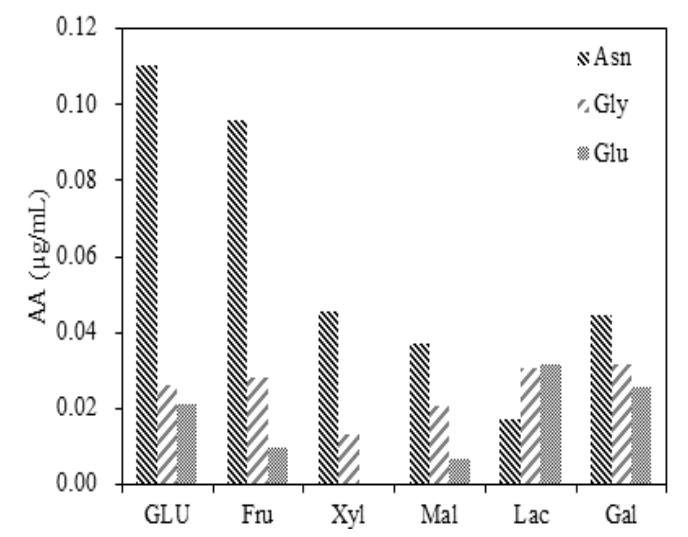

Figure 1. Acrylamide formation in different amino acid/reducing sugar model systems

\subsection{Dynamic of AA Formation in Asn/reducing Sugar Model Reaction Systems}

To further investigate the effects of reducing sugar on acrylamide formation, the dynamics of three types of reducing sugar (GLU, Fru and Xyl) with asparagine in model reaction system were studied.

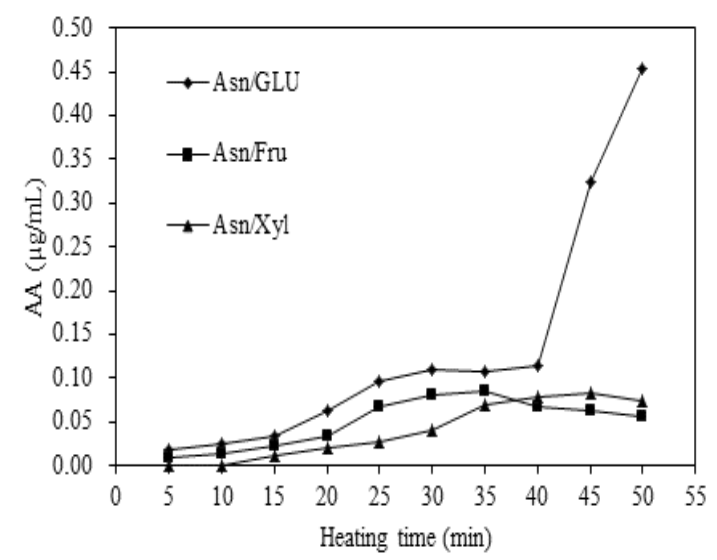

Figure 2. Acrylamide formation in three different dynamic asparagine/reducing sugar systems

As shown in Figure 2, the largest amount of AA among the three Asn/reducing sugar model systems was produced in the Asn/GLU dynamic system. In addition, the amount of AA increased with the extension of the heating time, and it reached a maximum at a certain heating time when the amount of AA decreased. For example, in the Asn/Fru system, the amount of AA increased with the extension of the heating time at $35 \mathrm{~min}$ from $0.0089 \mu \mathrm{g} / \mathrm{mL}$ to 0.0852 $\mu \mathrm{g} / \mathrm{mL}$, and then decreased to $0.0565 \mu \mathrm{g} / \mathrm{mL}$ in the next 15 min. Extraordinarily, there was a sudden increase of AA when the Asn/GLU model system was heated to an anhydrous state at $160^{\circ} \mathrm{C}$ for $40 \mathrm{~min}$, which indicated that more AA is generated when the Maillard reaction is reacted in an anhydrous state.

\subsection{Formation of AA in Mixed Amino Acid/GLU Model Systems}


As shown in Figure 3, different amounts of AA were produced when three amino acids added to the Asn/GLU model system. The abilities of these three amino-acids to produce AA in mixed amino acid/GLU model systems were ranked as follows: Gly $(0.0817 \mu \mathrm{g} / \mathrm{mL})>$ Glu $(0.0584 \mu \mathrm{g} / \mathrm{mL})>$ Cys $(0.0407 \mu \mathrm{g} / \mathrm{mL})$.

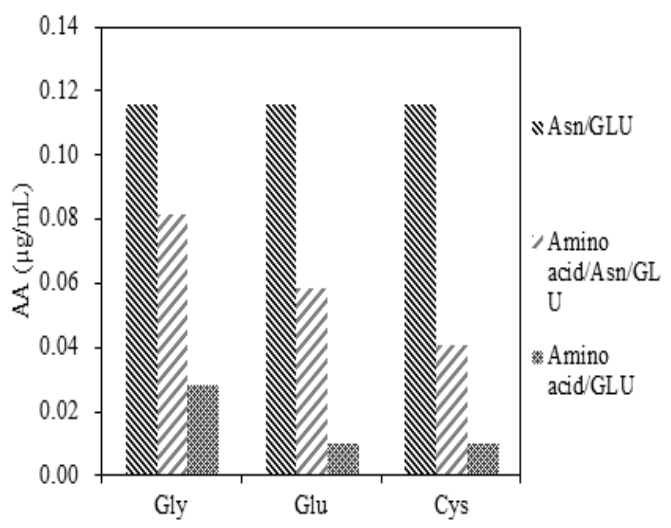

Figure 3. Formation of acrylamide in Asn/GLU model system, amino acid/GLU model systems and mixed amino acid/Asn/GLU model systems

Based on the amount of AA produced in the Asn/GLU model system, the addition of another type of amino acid reduced the formation of AA. The largest decrease in AA production (approximately 65\%) occurred in the Cys/ Asn/GLU model system, the second largest decrease in AA production (approximately 50\%) occurred in the Glu/Asn/GLU model system. Morever, the smallest decrease in AA production (approximately 30\%) occurred in the Gly/Asn/GLU model system. The amount of AA produced in the amino acid/Asn/GLU model systems was less than that produced in the Asn/GLU model system. However, the amount of AA produced was higher than that produced in the amino acid/GLU model system. From a mechanistic point of view, it was proposed that the amount of AA produced in Gly/Asn/GLU model system might be the average of that produced in the Asn/GLU model system and the Gly/GLU model system, but the amount was greater. Therefore, a complex competition might occur between Asn and other amino acids.

\subsection{Effects of Vitamin Additives on AA Formation}

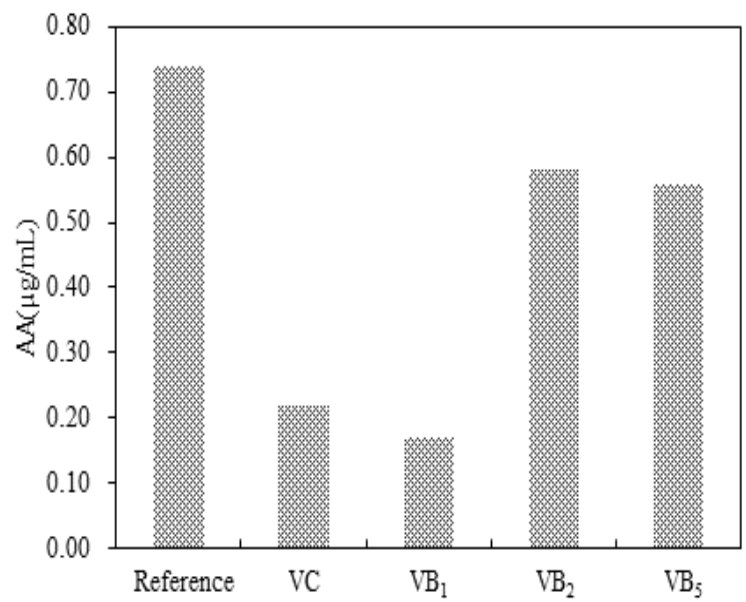

Figure 4. Effects of vitamin additives on the formation of acrylamide based on Asn/GLU model systems
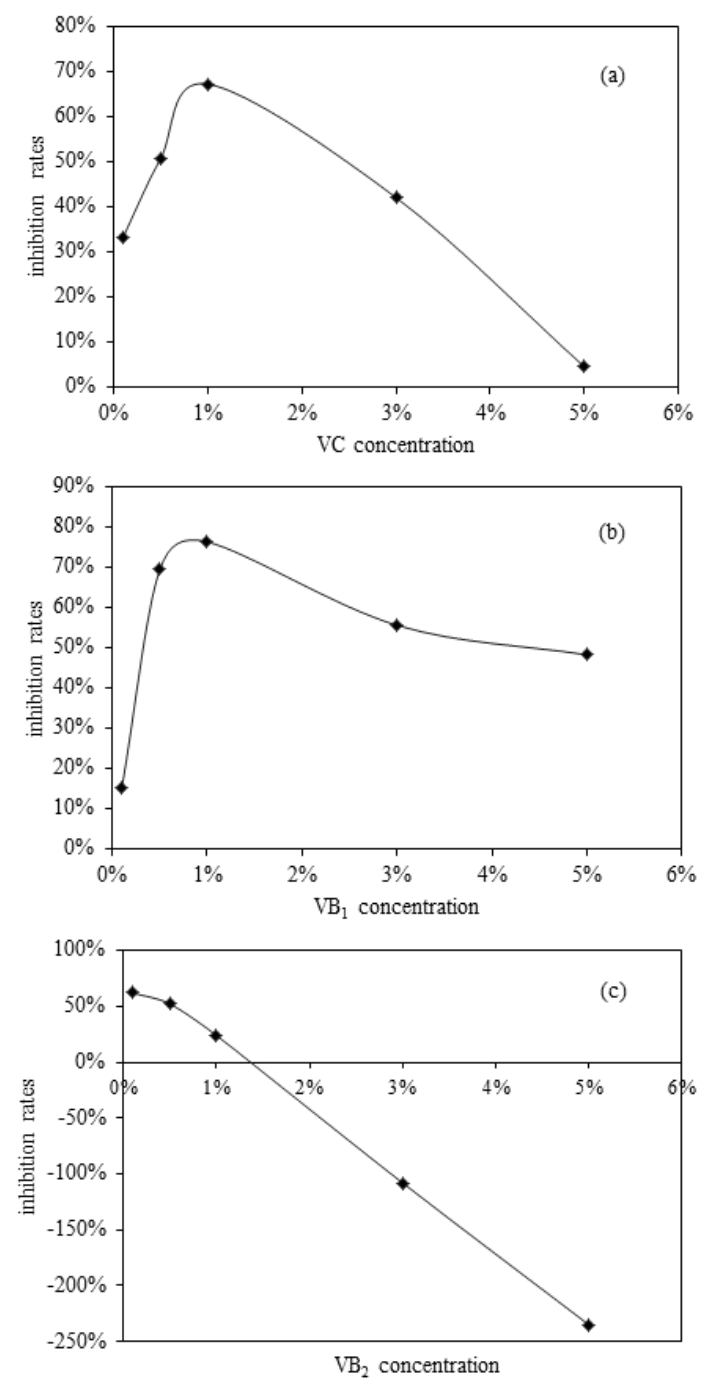

Figure 5. Inhibition rates of (a) VC, (b) VB1 and (c) VB2 to acrylamide formation based on Asn/GLU model system

Based on the amount of AA produced in the Asn/GLU model system, the formation of AA was reduced by all of the tested water-soluble vitamins as shown in Figure 4. $\mathrm{VC}$ and $\mathrm{VB}_{1}$ strongly reduced the formation of $\mathrm{AA}$ by more than $60 \%$, whereas $\mathrm{VB}_{2}$ and $\mathrm{VB}_{5}$ only reduced the amount of AA formed by approximately $20-30 \%$.

Different dosages of vitamins have different capacity to reduce the formation of $\mathrm{AA}$ as shown in Figure 5. Moreover, the inhibition rates of AA increased at the beginning followed by a reduction, and the rates peaked at approximately $1 \%$ under the adding concentration gradient of $0.1-5 \%$, as shown in Figure 5 (a) and Figure 5 (b). Therefore, to maximize the AA formation inhibitory capacity of vitamins in the model system, the appropriate adding dosage of $\mathrm{VC}$ and $\mathrm{VB}_{1}$ is approximately $1 \%$. However, the inhibition rates of AA were in a downward trend, and they turned negative at approximately $1 \%$ under the added concentration gradient of $0.1-5 \%$, as shown in Figure 5 (c). Consequently, the appropriate adding dosage of $\mathrm{VB}_{2}$ is approximately within $1 \%$, as $\mathrm{VB}_{2}$ promoted the formation of $\mathrm{AA}$ at adding dosages greater than $1 \%$.

\section{Conclusions}

In this study, the effect of amino acids, reducing sugars and vitamins on the formation of AA in the amino 
acid/sugar chemical model system was systematically investigated. The results showed that different amino acid/sugar mixtures have different abilities to produce AA, and all of the tested vitamins were capable of inhibiting AA production. The largest amount of AA was produced in the Asn/GLU model system among the tested amino acid/reducing sugar model systems. Gly, Glu and Cys caused different levels of reduction in the amounts of AA, which may be due to the different degree of competition of Asn with other amino acids in the mixed amino acid/GLU model systems. VC and $\mathrm{VB}_{1}$ strongly reduced the formation of AA by more than $60 \%$ at the appropriate adding dosage of approximately $1 \%$, whereas $\mathrm{VB}_{2}$ and $\mathrm{VB}_{5}$ only reduced the formation of AA by $20-30 \%$ at the adding dosage of approximately $1 \%$. In contrast, $\mathrm{VB}_{2}$ promoted the formation of AA at adding dosages greater than $1 \%$. Further studies are required to characterize the action mechanism of the vitamin additives that showed strong inhibitory effect against the formation of AA.

\section{Acknowledgements}

This work was carried out with support from AQSIQ (General Administration of Quality Supervision), Special Scientific Research Fund of public welfare profession for quality inspection, No. 201210016.

\section{Statement of Competing Interests}

The authors have no competing interests.

\section{References}

[1] Swedish National Food Administration. 2002. Information About Acrylamide in Food http://www.slv.se (accessed Feb 23, 2013).

[2] Ahn, J. S., Castle, L., Clarke, D. B., Lloyd, A. S., Philo, M. R. and Speck, D. R. 2002. Verification of the findings of acrylamide in heated foods. Food Additives and Contaminants, 19, 1116-1124.

[3] Tareke, E., Rydberg, P., Karlsson, P. Eriksson, S. and Tornqvist, M. 2002. Analysis of acrylamide, a carcinogen formed in heated foodstuffs. Journal of Agricultural and Food Chemistry, 50, 49985006.

[4] Becalski, A., Lau, B. P-Y., Lewis, D., and Seaman, S. W. 2003. Acrylamide in food: Occurrence, sources, and modeling. Journal of Agricultural and Food Chemistry, 51, 802-808.

[5] Friedman, M. 2003. Chemistry, biochemistry, and safety of acrylamide. Journal of Agricultural and Food Chemistry, 51, 4504-4526.

[6] Friedman, M. and Levin, C.E. 2008. Review of methods for the reduction of dietary content and toxicity of acrylamide. Journal of Agricultural and Food Chemistry, 56, 6113-6140.

[7] Mottram, D.S. Wedzicha, B.L. and Dodson, A.T. 2002. Acrylamide is formed in the Maillard reaction. Nature, 419, 448449.

[8] Stadler, R.H. Blank, I., Varga, N., Robert, F., Hau, J., Guy, P.A., Robert, M.C. and Riediker, S. 2002. Acrylamide from Maillard reaction products. Nature. 419, 449-450.

[9] Yaylayan, V.A., Wnorowski, A. and Locas, C.P. 2003. Why asparagine needs carbohydrates to generate acrylamide. Journal of Agricultural and Food Chemistry, 51, 1753-1757.

[10] Blank, I., Robert, F., Goldmann, T., Pollien, P., Varga, N., Devaud, S., Saucy, F., Huynh-Ba, T. and Stadler, RH. 2005. Mechanisms of acrylamide formation: Maillard-induced transformation of asparagine. Advances in Experimental Medicine and Biology, 561, 171-189.

[11] Zhang, Y. and Zhang, Y. 2007. Formation and reduction of acrylamide in Maillard reaction: a review based on the current state of knowledge. Critical Reviews in Food Science and
Nutrition, 47, 521-542. Becalski, A., Lau, B. P-Y., Lewis, D., Seaman, S.W., Hayward, S., Sahagian, M., Ramesh, M. and Leclerc, Y. 2004. Acrylamide in French Fries: Influence of Free Amino Acids and Sugars. Journal of Agricultural and Food Chemistry, 52, 3801-3806.

[12] Becalski, A., Lau, B. P-Y., Lewis, D., Seaman, S.W., Hayward, S., Sahagian, M., Ramesh, M. and Leclerc, Y. 2004. Acrylamide in French Fries: Influence of Free Amino Acids and Sugars. Journal of Agricultural and Food Chemistry, 52, 3801-3806.

[13] Koutisidis, G., Simons, S.P.J., Thong, Y.H., Haldoupis, Y., Mojica-Lazaro, J., Wedzicha, B.L. and Mottram, D.S. 2009. Investigations on the effect of amino acids on acrylamide, pyrazines, and Michael addition products in model systems. Journal of Agricultural and Food Chemistry, 57, 9011-9015.

[14] Zamoria, Delgado, R. and Hidalgo, FJ. 2010. Model reactions of acrylamide with selected amino compounds. Journal of Agricultural and Food Chemistry, 58, 1708-1713.

[15] Liu, J., Chen, F., Man, Y., Dong, L. and Hu, X.S. 2011. The pathways for the removal of acrylamide in model systems using glycine based on the identification of reaction products. Food Chemistry, 128, 442-449.

[16] Jung, M.Y., Choi, D.S. and Ju, J.W. 2003. A novel technique for limitation of acrylamide formation in fried and baked corn chips and in French fries. Journal of Food Science, 68, 1287-1290.

[17] Pedreschi, F., Granby, K. and Risum, J. 2010. Acrylamide mitigation in potato chips by using $\mathrm{NaCl}$. Food and Bioprocess Technology, 3, 917-921.

[18] Mestdagh, F., Maertens, J., Cucu T., Delporte, K., Van Peteghem, C. and De Meulenaer, B. 2008. Impact of additives to lower the formation of acrylamide in a potato model system through $\mathrm{pH}$ reduction and other mechanisms. Food Chemistry, 107, 26-31.

[19] Casado, F.J., Sánchez, AH. and Montaño, A. 2010. Reduction of acrylamide content of ripe olives by selected additives. Food Chemistry, 119, 161-166.

[20] Cheng, K.W., Zeng, X., Tang, Y.S., Wu, J.J., Liu, Z.W., Sze, K.H. Chu, I.K., Chen, F. and Wang, M.F. 2009. Inhibitory mechanism of naringenin against carcinogenic acrylamide formation and nonenzymatic browning in Maillard model reactions. Chemical Research in Toxicology, 22, 1483-1489.

[21] Bassama, J., Brat, P., Bohuon, P., Boulangera, R. and Günata, Z. 2010. Study of acrylamide mitigation in model system: Effect of pure phenolic compounds. Food Chemistry, 123, 558-562.

[22] Zhang, Y., Xu, W.Z., Wu, X.Q., Zhang, X.L. and Zhang, Y. 2007. Addition of antioxidant from bamboo leaves as an effective way to reduce the formation of acrylamide in fried chicken wings. Food Additives and Contaminants, 24, 242-251.

[23] Cheng, K.W., Shi, J.J., Ou, S.Y., Wang, M.F. and Jiang, Y. 2010. Effects of fruit extracts on the formation of acrylamide in model reactions and fried potato crisps. Journal of Agricultural and Food Chemistry, 58, 309-312.

[24] Geng, Z., Jiang, R. and Chen, M. 2009. Effects of Dough Formula and Technology Condition on the Formation of Acrylamide in Shaobing. Journal of Chinese Institute of Food Science and Technology, 9, 143-148.

[25] Tareke, E. 2003. Identification and origin of potential background carcinogens: Endogenous isoprene and oxiranes, dietary acrylamide. Department of Environmental Chemistry, Stockholm University.

[26] Gökmen, V. and Şenyuva, H.Z. 2007. A simplified approach for the kinetic characterization of acrylamide formation in fructoseasparagine model system. Food Additives and Contaminants, 23, 348-354.

[27] Rasim, A. O., Mahmut, D., and Kemal, S. 2014. Effects of certain polyphenols and extracts on furans and acrylamide formation in model system, and total furans during storage. Food Chemistry, $142,423-429$.

[28] Rosén, J. and Hellenäs, K.E. 2002. Analysis of acrylamide in cooked foods by liquid chromatography tandem mass spectrometry. The Analyst, 127, 880-882.

[29] Zhuang, H., Zhang, T.H., Liu, J.B. and Yuan, Y. 2012. Detection of acrylamide content in traditional Chinese food by highperformance liquid chromatography tandem mass spectrometry method. CyTA - Journal of Food, 10, 36-41. 